PAPER

Rotation analysis on large complex superconducting cables based on numerical modeling and experiments

To cite this article: Jinggang Qin et al 2018 Supercond. Sci. Technol. 31025001

View the article online for updates and enhancements.
Related content

- Optimization of CFETR CSMC cabling
$\frac{\text { based on numerical modeling and }}{\text { experiments }}$
$\frac{\text { Jinggang Qin, Chao Dai, Bo Liu et al. }}{\text { - New design of cable-in-conduit conductor }}$
$\frac{\text { for application in future fusion reactors }}{\text { Jinggang Qin, Yu Wu, Jiangang Li et al. }}$
- Cable-in-conduit conductors: lessons from
the recent past for future developments
with low and high temperature
superconductors
L Muzzi, G De Marzi, A Di Zenobio et al.




\title{
Rotation analysis on large complex superconducting cables based on numerical modeling and experiments
}

\author{
${\text { Jinggang } \text { Qin }^{1}{ }^{\oplus} \text {, Donghua Yue }}^{2,3}$, Xingyi Zhang ${ }^{2,3}, \mathbf{Y u} \mathbf{W u}^{1}$, \\ Xiaochuan Liu ${ }^{1}$, Huajun Liu ${ }^{1}$, Huan Jin ${ }^{1}$, Chao Dai ${ }^{1}$, Arend Nijhuis ${ }^{4}$, \\ Chao Zhou ${ }^{4}$ and Arnaud Devred

\footnotetext{
${ }^{1}$ Institute of Plasma Physics, Chinese Academy of Sciences, Hefei, Anhui, 230031, People's Republic of China

${ }^{2}$ Key Laboratory of Mechanics on Disaster and Environment in Western China attached to the Ministry of Education of China, Lanzhou University, Lanzhou, Gansu 730000, People's Republic of China

${ }^{3}$ Department of Mechanics and Engineering Sciences, College of Civil Engineering and Mechanics, Lanzhou University, Lanzhou, Gansu 730000, People's Republic of China

${ }^{4}$ University of Twente, Energy, Materials and Systems, Faculty of Science and Technology, Enschede, 7500AE, Netherlands
} \\ ${ }^{5}$ CERN, TE Department, Geneva 23 1211, Switzerland \\ E-mail: zhangxingyi@1zu.edu.cn and liuhj@ipp.ac.cn
}

Received 16 September 2017, revised 20 November 2017

Accepted for publication 22 November 2017

Published 19 December 2017

\begin{abstract}
The conductors used in large fusion reactors, e.g. ITER, CFETR and DEMO, are made of cable-inconduit conductor (CICC) with large diameters up to about $50 \mathrm{~mm}$. The superconducting and copper strands are cabled around a central spiral and then wrapped with stainless-steel tape of $0.1 \mathrm{~mm}$ thickness. The cable is then inserted into a jacket under tensile force that increases with the length of insertion. Because the cables are long and with a large diameter, the insertion force could reach values of about $40 \mathrm{kN}$. The large tensile force could lead to significant rotation forces. This may lead to an increase of the twist pitch, especially for the final one. Understanding the twist pitch variation is very important; in particular, the twist pitch of a cable inside a CICC strongly affects its properties, especially for $\mathrm{Nb}_{3} \mathrm{Sn}$ conductors. In this paper, a simplified numerical model was used to analyze the cable rotation, including material properties, cabling tension as well as wrap tension. Several rotation experiments with tensile force have been performed to verify the numerical results for CFETR CSMC cables. The results show that the numerical analysis is consistent with the experiments and provides the optimal cabling conditions for large superconducting cables.
\end{abstract}

Keywords: cable rotation, numerical model, tensile experiment, performance degradation

(Some figures may appear in colour only in the online journal)

\section{Introduction}

The magnet system of the International Thermonuclear Experimental Reactor (ITER), China Fusion Engineering Test Reactor (CFETR), and DEMO (EU-DEMO, K-DEMO) are composed of toroidal field (TF) coils, poloidal field coils, a central solenoid (CS), and a set of correction coils [1-3]. The superconductors for the TF and CS coils are manufactured from $\mathrm{Nb}_{3} \mathrm{Sn}$ strands [4]. Generally, the conductor consists of five cabling stages around a central cooling spiral. Final cabling uses six sub-cables (petals) consisting of $\mathrm{Nb}_{3} \mathrm{Sn}$ strands and copper strands. Eventually, the cable has more than 1000 strands, e.g. ITER TF has $900 \mathrm{Nb}_{3} \mathrm{Sn}$ and 522 copper strands, shown in figure 1 [5]. To form a cable-inconduit conductor (CICC), a cable is inserted into a jacket under tensile force [6-9]. During cable insertion, several tons of tension is generated by the friction between cable and jacket. For the Chinese ITER TF cable production, the 

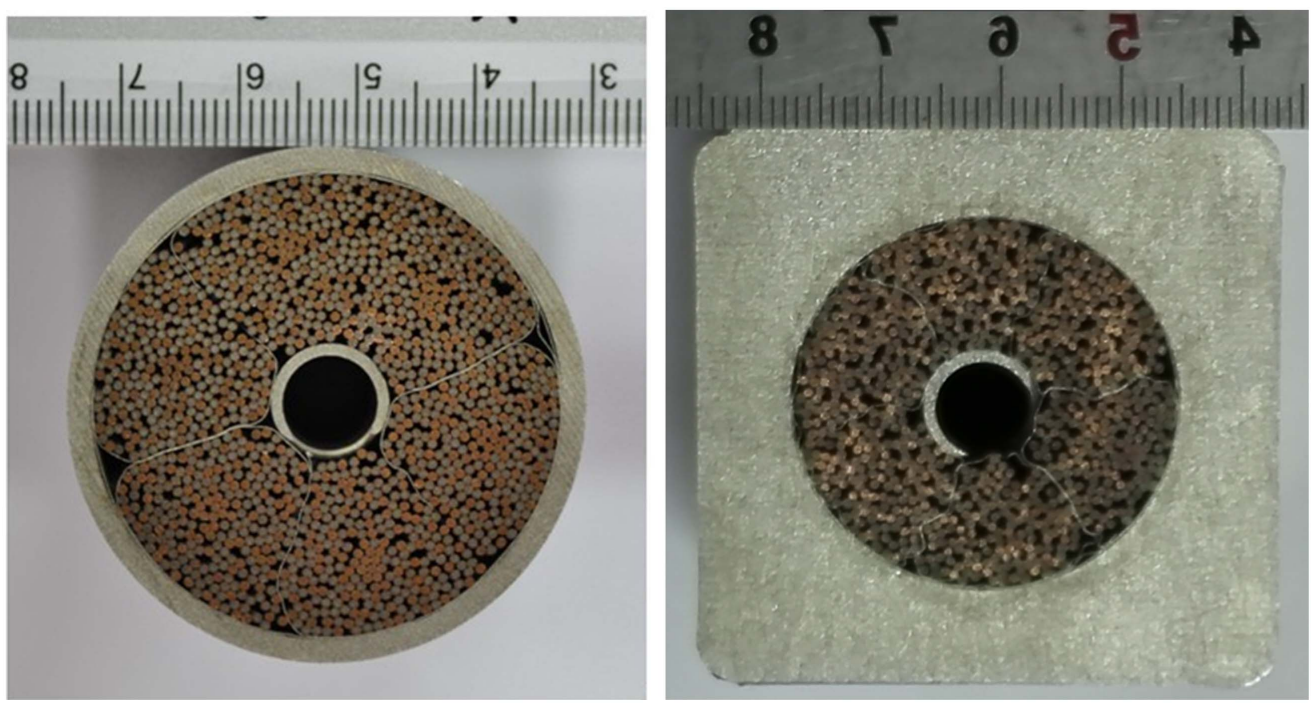

Figure 1. Cross-section of conductor (left: ITER TF, right: CFETR CSMC).

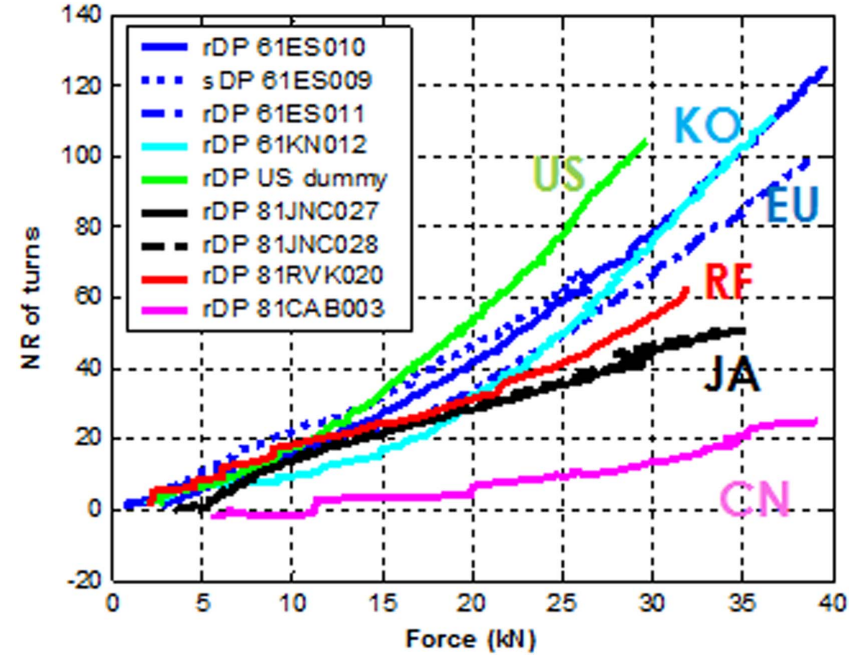

Figure 2. The compared TF cable rotation.

maximum insertion force could reach to $40 \mathrm{kN}$ [8]. In the future, the dimensions of cables used for the CFETR or DEMO could be even larger than the present ones for ITER $[10,11]$, which would require a much higher insertion force than that used for the ITER cables.

This tension can lead to both stretching and untwisting of a cable resulting in an increase of the twist pitch for the last stage of cabling. The resultant twist pitches have exceeded the specified allowable twist pitch according to the requirements [4]. The varied twist pitch measured at high performance magnetic (HPM) for ITER TF changed from 420 to $525 \mathrm{~mm}$ along the full cable length $(760 \mathrm{~m})$, which increased by $25 \%$ [12]. In JAEA, the cable twist pitch of ITER TF and CS increased by about $15 \%$ and $20 \%$ along the conductor length (TF-760 m, CS-918 m), respectively [13, 14]. In Russian Scientific R\&D Cable Institute (VNIIKP), the cable twist pitch of ITER TF increased by about $9 \%$ [15]. The allowable value of increased twist pitch is less than 5\% for ITER TF and $\mathrm{CS}$, respectively.

Firstly, twist pitch elongation may result in an increase of AC loss [16]. Each sub-cable is wrapped separately with a stainless-steel sheet covering around $50 \%-70 \%$ of its out surface area. As a result, coupling currents induced by an applied alternating magnetic field are expected to be restricted essentially to each sub-cable. Regarding to a prototype CS baseline conductor made in 2001, experiments suggested that the AC coupling current were mainly limited to each subcable as long as the CICC void fraction exceeded 25\% [17]. This conclusion should be also applicable to the ITER TF and $\mathrm{CS}$, as well as some future conductors, since all the void fractions exceed the value $[10,11]$.

Secondly, the increased twist pitch could reduce the cable stiffness, thus reduce mechanical support among sub-cables. During cycling of electromagnetic force and warm-up-cooldown (WUCD), the variation in current sharing temperature $\left(T_{\mathrm{cs}}\right)$ could appear. For the $T_{\mathrm{cs}}$ characteristics of CS conductors established by the SULTAN facility, a clear degradation for conductor with enlarged twist pitch was not observed [18]. However, a systematic investigation is needed to evaluate the impact of cable twist pitch elongation on $T_{\mathrm{cs}}$ degradation. As learned from ITER CS short twist design, shorter twist pitch enhances the cable stiffness, suppresses the movement of individual strands and $T_{\mathrm{cs}}$ degradation due to EM cycling and WUCD [4, 7, 18, 19].

Furthermore, it is clear that the increased twist pitch could increase the void fraction, which might locally make a space large enough leading to strand movements. Consequently, uncertainties on conductor performance and thermal stability could appear due to the cable twist pitch elongation, and more experiments and analysis are required to understand the influence of cable untwisting, in terms of CICC mechanical property, $T_{\mathrm{cs}}$, and thermal stability. The cable twist pitches in CICCs should be well controlled in the required range. 


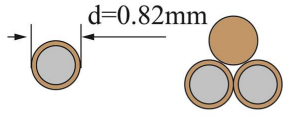

$\mathrm{Nb} 3 \mathrm{Sn}$ wire $\left(2^{*} \mathrm{Nb} 3 \mathrm{Sn}+\mathrm{Cu}\right)$ strand

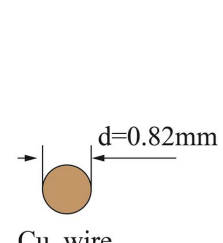

$\mathrm{Cu}$ wire

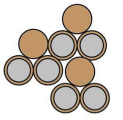

$3 * 3$ strand

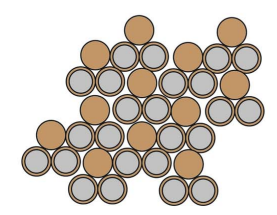

$3 * 3 * 4$ strand

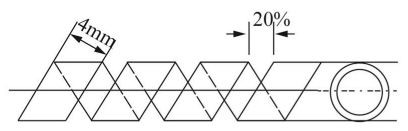

Central spiral $1 \mathrm{~mm}$ thick
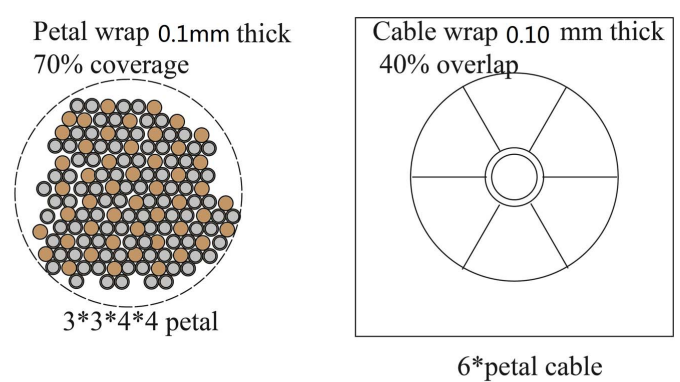

Figure 3. The dimensions and parameters of a model cable (e.g. CFETR CSMC).

Table 1. Parameters of the CFETR CSMC cable.

\begin{tabular}{ll}
\hline Item & Parameter \\
\hline $\mathrm{Nb}_{3}$ Sn strand & $0.82 \pm 0.005 \mathrm{~mm}$ \\
Copper strand & $0.82 \pm 0.005 \mathrm{~mm}$ \\
Layout & $((2 \mathrm{Sc}+1 \mathrm{Cu}) \times 3 \times 4 \times 4) \times 6$ \\
Pitch $(\mathrm{mm})$ & \\
Stage 1 & $25 \pm 1$ \\
Stage 2 & $45 \pm 8$ \\
Stage 3 & $80 \pm 10$ \\
Stage 4 & $150 \pm 15$ \\
Stage 5 & $450 \pm 20$ \\
Twist direction & Right hand \\
Sub-wrap (mm) & $0.1 \times 15$ \\
Sub-wrap coverage & $70 \pm 5 \%$ \\
Cable diameter (mm) & $33.5+0.2-0.3$ \\
Wrap (mm) & $0.1 \times 40$ \\
Wrap overlap & $30 \%-40 \%$ \\
Central cooling tube (mm) & $10 \times 1$ \\
\hline
\end{tabular}

In order to better understand the untwisting and final stage twist pitches, experiments have been performed at HPM (USA), JAEA (Japan), VNIIKP (Russia), and ASIPP (China) to evaluate the cable rotation of their ITER TF or CS cables $[12-15,20]$, shown in figure 2 . They developed devices to monitor the cable rotation during insertion and tested some short cable samples under tension. In figure 2, it is shown that the number of rotations can be very different for the same type of cable depending on the manufacturer [21], which suggests that some cabling parameters are different.

This paper aims at analyzing the causes of cable rotation during insertion and at optimizing the cabling technique to reduce the cable rotation by the combination of numerical modeling and experiments. The numerical model describes the rotational behavior under tension. The stiffness of the petal was modeled firstly with the parameters axial tension, sub-wrap tension, curvature and friction. The final cable was then simplified to the six petals, central cooling tube and cable wrap. The final cabling tension, wrapping tension, pitch and friction among strands were considered as main parameters in the model. Based on the numerical results, different cabling experiments were selected and performed. Four CFETR CSMC type dummy cables were manufactured with different cabling parameters. The cable rotation of each cable was tested under force, which reached to $40 \mathrm{kN}$. The rotation shows different behavior depending on the different cabling parameters. The experimental results were compared to the numerical analysis, showing good agreement. An important observation is that a large cable wrap tension reduces the cable rotation effectively. The final cabling tension has less influence on the cable rotation. The model can be used to estimate and optimize the cabling parameters, and keep the increased twist pitch in the required range, which could keep the conductor performance in stable level. The results are very beneficial for the upcoming manufacture of the large cables for CFETR and DEMO.

\section{Numerical model}

In this section, the numerical model for a large-scale cable, as used in e.g. ITER TF and CFETR CSMC, is described. The components of the cable in the model include petals, central cooling spiral, and wrap, as shown schematically in figure $3[5,21]$.

The model ignores the friction between the jacket and cable and only accounts for the de-twisting of the cable under the insertion force $F_{\text {Insert }}$.

The twist direction of the central cooling spiral and cable wrap is left and that of the petals is right. There is no torsion constraint for the cable when it is under free axial stretch. The boundary conditions are set as $F=F_{0}$ and $M=0$.

The forces on the whole cable is from those acting on the central cooling spiral, sub-cables and wrap, which can be expressed as:

$$
\begin{aligned}
& F=F_{\text {in }}+F_{\mathrm{p}}+F_{\mathrm{st}}=F_{0}, \\
& M=M_{\text {in }}+M_{\mathrm{p}}+M_{\mathrm{st}}=0 .
\end{aligned}
$$




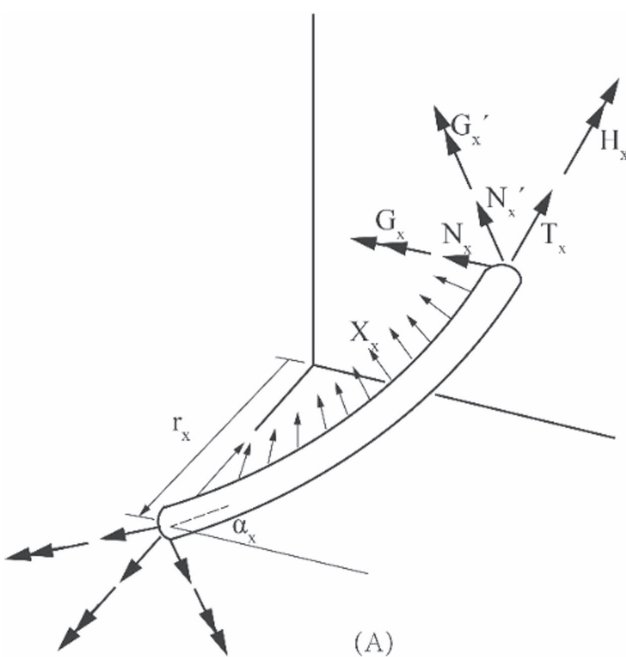

(A)

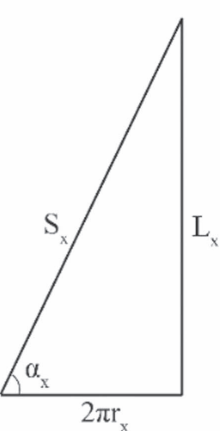

(B)

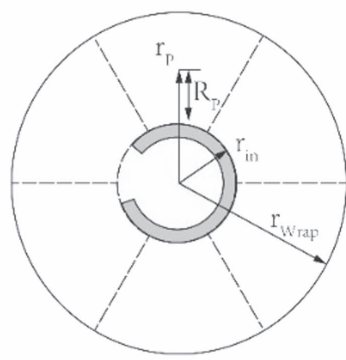

(C)

Figure 4. Force distribution (left) and spatial relationship (B) and (C).

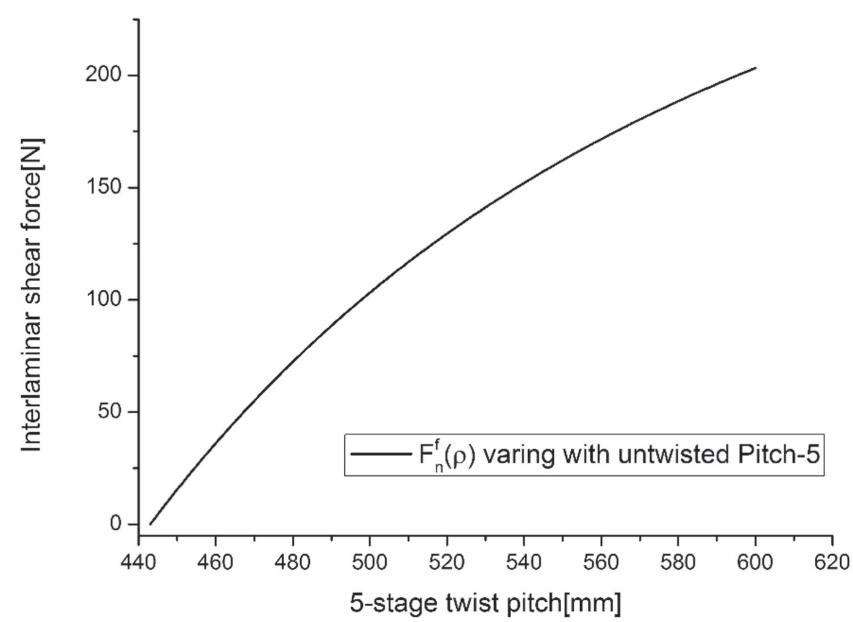

Figure 5. The relationship between interlayer shear force and detwisted pitch.

In the equations, $F$ refers to the axial insertion force for the whole cable. $F_{\text {in }}, F_{\mathrm{p}}, F_{\mathrm{st}}$ are the forces acting on the central cooling spiral, sub-cables and wrap, respectively. $M$ is the torque on the whole cable. $M_{\text {in }}, M_{\mathrm{p}}, M_{\mathrm{st}}$ are the torques of central cooling spiral, petals and wrap. The axial elongation and torsion deformation of the cable are expressed as $\varepsilon_{0}, \beta_{0}$.

The force distribution and spatial relationship are shown in figure 4. $G_{x}, G_{x}^{\prime}, H_{x}$ are sectional moment components defined as thin rods. $N_{x}, N_{x}^{\prime}, T_{x}$ are the sectional force components. $X_{x}$ is the contact force and $\alpha_{x}$ is the spiral angle of each component. $r_{x}$ is the distance between the centroid and the center of the cable, and $L_{x}$ is the twist pitch.

The central cooling spiral is modeled as a single helical spiraled thin strip. The axial strain, change of radius and spiral angle change are defined as $\xi_{\mathrm{st}}, \frac{\Delta r_{\mathrm{st}}}{r_{\mathrm{st}}}, \Delta \alpha_{\mathrm{st}}$, respectively.

According to Costello-Velinsky's theory [22-24], the geometrical equations of the central cooling spiral, petals, and wrap are expressed as:

$$
\begin{aligned}
& \text { He-inlet } \quad:\left\{\begin{array}{l}
\beta_{0}=\frac{\xi_{\text {in }}}{\tan \alpha_{\text {in }}}+\frac{1}{\tan \alpha_{\text {in }}} \frac{\Delta r_{\text {in }}}{r_{\text {in }}}-\Delta \alpha_{\text {in }} \\
\text { Petal }
\end{array} \quad \begin{array}{c}
\varepsilon_{\mathrm{P}}+\frac{\Delta \alpha_{\mathrm{P}}}{\tan \alpha_{\mathrm{P}}} \\
\beta_{0}=\frac{\xi_{\mathrm{P}}}{\tan \alpha_{\mathrm{P}}}+\frac{1}{\tan \alpha_{\mathrm{P}}} \frac{\Delta r_{\mathrm{P}}}{r_{\mathrm{P}}}-\Delta \alpha_{\mathrm{P}}
\end{array}\right. \\
& \text { Wrap-stiffness }:\left\{\begin{array}{c}
\varepsilon_{0}=\xi_{\mathrm{st}}+\frac{\Delta \alpha_{\mathrm{st}}}{\tan \alpha_{\mathrm{st}}} \\
\beta_{0}=\frac{\xi_{\mathrm{st}}}{\tan \alpha_{\mathrm{st}}}+\frac{1}{\tan \alpha_{\mathrm{st}}} \frac{\Delta r_{\mathrm{st}}}{r_{\mathrm{st}}}-\Delta \alpha_{\mathrm{st}} .
\end{array}\right.
\end{aligned}
$$$$
:\left\{\begin{array}{c}
\varepsilon_{0}=\xi_{\text {in }}+\frac{\Delta \alpha_{\text {in }}}{\tan \alpha_{\text {in }}} \\
\beta_{0}=\frac{\xi_{\text {in }}}{\tan \alpha_{\text {in }}}+\frac{1}{\tan \alpha_{\text {in }}} \frac{\Delta r_{\text {in }}}{r_{\text {in }}}-\Delta \alpha_{\text {in }}
\end{array}\right.
$$

In the equations, only the axial tension was considered for the central cooling spiral and wrap. Therefore, the equations can be adapted as:

$$
\begin{aligned}
& \text { He-inlet: }\left\{\begin{array}{c}
G_{\text {in }}=0 ; G_{\text {in }}^{\prime}=0 ; H_{\text {in }}=0 \\
T_{\text {in }}=E_{\text {in }} A_{\text {in }} \xi_{\text {in }}
\end{array}\right. \\
& \text { Petal: }\left\{\begin{array}{c}
G_{\mathrm{P}}=0 ; G_{\mathrm{P}}^{\prime}=E_{\mathrm{P}} I_{\mathrm{P}}^{x} \Delta \kappa_{\mathrm{P}}^{\prime} ; H_{\mathrm{P}}=G_{\mathrm{P}} I_{\mathrm{P}}^{z} \Delta \tau_{\mathrm{P}} \\
T_{\mathrm{P}}=E_{\mathrm{P}} A_{\mathrm{P}} \xi_{\mathrm{P}}
\end{array}\right. \\
& \text { Wrap-stiffness: }\left\{\begin{array}{c}
G_{\mathrm{st}}=0 ; G_{\mathrm{st}}^{\prime}=0 ; H_{\mathrm{st}}=0 \\
T_{\mathrm{st}}=E_{\mathrm{st}} A_{\mathrm{st}} \xi_{\mathrm{st}} .
\end{array}\right.
\end{aligned}
$$

In the equation system, $E$ refers to the young's modulus of each component and $A$ refers to the cross-section of each component. $E_{\mathrm{P}} I_{\mathrm{P}}^{x}, \quad G_{\mathrm{P}} I_{\mathrm{P}}$ are the bending and torsion stiffness of the petals, respectively. $\Delta \kappa_{\mathrm{P}}^{\prime}, \Delta \tau_{\mathrm{P}}$ represent the changes of curvature and twist, respectively. $\xi_{\mathrm{p}}$ is the axial strain of petal.

The balance equations of the central cooling spiral, subcables and wrap can be formulated as:

$$
\begin{aligned}
N_{x}^{\prime}= & G_{x}^{\prime} \tau_{x}+H_{x} \kappa_{x}^{\prime}, \\
X_{x}= & N_{x}^{\prime} \tau_{x}-T_{x} \kappa_{x}^{\prime}, \\
F_{x}= & T_{x} \sin \alpha_{x}+N_{x}^{\prime} \cos \alpha_{x}, \\
M_{x}= & H_{x} \sin \alpha_{x}+G_{x}^{\prime} \cos \alpha_{x}+T_{x} r_{x} \cos \alpha_{x} \\
& \quad-N_{x}^{\prime} r_{x} \sin \alpha_{x},
\end{aligned}
$$




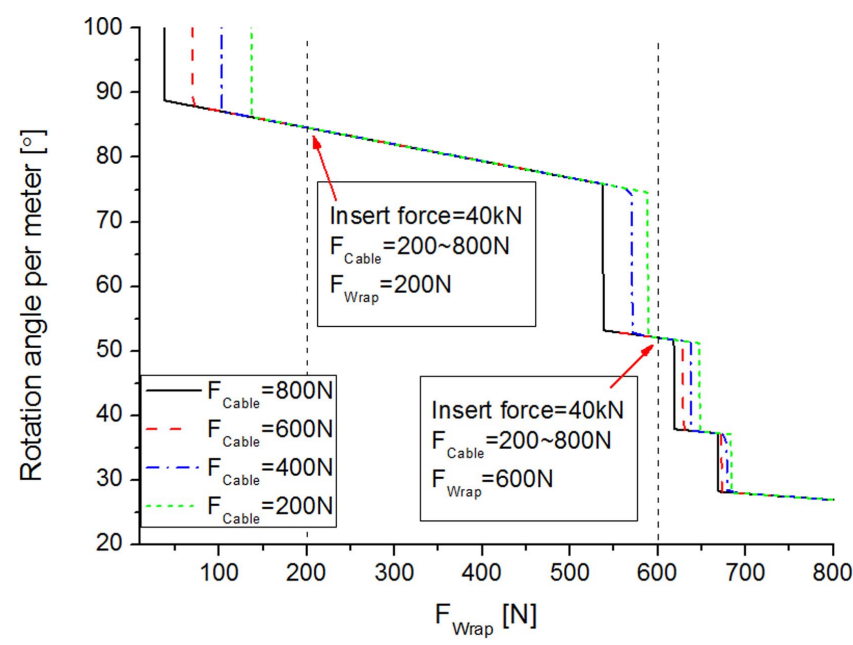

Figure 6. The cable rotation under different conditions.

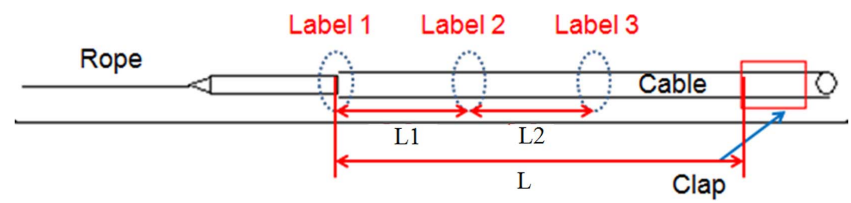

Figure 7. Scheme of the positions for the rotation measurement and recording.

Table 2. Cabling experiments with different parameters.

\begin{tabular}{lcc}
\hline Sample & Final cabling tension $(\mathrm{N})$ & Final wrapping tension $(\mathrm{N})$ \\
\hline CS-1 & 200 & 600 \\
CS-2 & 800 & 600 \\
CS-3 & 200 & 200 \\
CS-4 & 800 & 200 \\
\hline
\end{tabular}

$$
\begin{aligned}
r_{\text {in }} & =R_{\text {in }} ; r_{\mathrm{P}}=R_{\text {in }}+R_{\mathrm{P}} ; r_{\mathrm{st}}=R_{\text {in }}+2 R_{\mathrm{P}}, \\
\Delta r_{\text {in }} & =\Delta R_{\text {in }} ; \Delta r_{\mathrm{P}}=\Delta R_{\text {in }}+\Delta R_{\mathrm{P}} ; \\
\Delta r_{\mathrm{st}} & =\Delta R_{\text {in }}+2 \Delta R_{\mathrm{P}}
\end{aligned}
$$

where $x$ is in, $p$, st, which represent central cooling spiral, petal and wrap, respectively. $R_{\mathrm{p}}$ is the twist radius of the petal and $\Delta R_{\mathrm{P}}=-\nu R_{\mathrm{P}} \xi_{\mathrm{P}}$, with $v$ being the Poisson's ratio of the sub-cable.

The equation of the contact force can be described as follows:

$$
\frac{6 X_{\mathrm{P}}}{\sin \alpha_{\mathrm{P}}}=\frac{X_{\mathrm{st}}}{\sin \alpha_{\mathrm{st}}}-\frac{X_{\mathrm{in}}}{\sin \alpha_{\mathrm{in}}},
$$

where $X_{\mathrm{P}}$ is the line pressure between petal and central cooling spiral. $X_{\mathrm{st}}$ is the line pressure between wrap layer and petal. $X_{\text {in }}$ is the support force between central cooling tube and petal.
From the above equations (1)-(4) we can obtain:

$$
\begin{aligned}
F= & F_{0}=6\left(T_{\mathrm{P}} \sin \alpha_{\mathrm{P}}+N_{\mathrm{P}}^{\prime} \cos \alpha_{\mathrm{P}}\right) \\
& +T_{\mathrm{st}} \sin \alpha_{\mathrm{st}}+T_{\mathrm{in}} \sin \alpha_{\mathrm{in}}, \\
M= & 0=6\left(H_{\mathrm{P}} \sin \alpha_{\mathrm{P}}+G_{\mathrm{P}}^{\prime} \cos \alpha_{\mathrm{P}}+T_{\mathrm{P}} r_{\mathrm{P}}\right. \\
& \left.\times \cos \alpha_{\mathrm{P}}-N_{\mathrm{P}}^{\prime} r_{\mathrm{P}} \sin \alpha_{\mathrm{P}}\right)-T_{\mathrm{st}} r_{\mathrm{st}} \cos \alpha_{\mathrm{st}} \\
& -T_{\mathrm{in}} r_{\mathrm{in}} \cos \alpha_{\mathrm{in}} .
\end{aligned}
$$

From equations (2)-(7) we can formulate:

$$
\begin{aligned}
& \left(\frac{1}{\tan \alpha_{\mathrm{st}}}+\tan \alpha_{\mathrm{p}}\right) \xi_{\mathrm{st}}-\frac{v r_{\mathrm{p}}}{\tan \alpha_{\mathrm{st}}} \frac{2}{r_{\mathrm{in}}+2 R_{\mathrm{P}}} \xi_{\mathrm{P}}+0 \cdot \xi_{\text {in }} \\
& +\frac{1}{\tan \alpha_{\mathrm{st}}} \frac{1}{r_{\mathrm{in}}+2 R_{\mathrm{P}}} \Delta r_{\mathrm{in}}-\tan \alpha_{\mathrm{st}} \varepsilon_{0}-\beta_{0}=0 \\
& 0 \cdot \xi_{\mathrm{st}}+\left(\frac{1}{\tan \alpha_{\mathrm{p}}}+\tan \alpha_{\mathrm{p}}-\frac{v R_{\mathrm{P}}}{\tan \alpha_{\mathrm{P}}} \frac{1}{r_{\mathrm{in}}+R_{\mathrm{P}}}\right) \xi_{\mathrm{P}} \\
& +0 \cdot \xi_{\text {in }}+\frac{1}{\tan \alpha_{\mathrm{P}}} \frac{1}{r_{\text {in }}+R_{\mathrm{P}}} \Delta r_{\text {in }}-\tan \alpha_{\mathrm{P}} \varepsilon_{0}-\beta_{0}=0 \\
& 0 \cdot \xi_{\text {st }}+0 \cdot \xi_{\mathrm{P}}+\left(\tan \alpha_{\text {in }}+\frac{1}{\tan \alpha_{\text {in }}}\right) \xi_{\text {in }} \\
& +\frac{1}{r_{\text {in }} \tan \alpha_{\text {in }}} \Delta r_{\text {in }}-\tan \alpha_{\text {in }} \varepsilon_{0}-\beta_{0}=0 \\
& -\frac{\sin \alpha_{\mathrm{P}}}{6 \sin \alpha_{\mathrm{st}}}(E A)_{\mathrm{st}} \kappa_{\mathrm{st}}^{\prime} \xi_{\mathrm{st}}+\left[G B \tau_{\mathrm{P}}+H B \kappa_{\mathrm{P}}^{\prime}-(E A)_{\mathrm{P}} \kappa_{\mathrm{P}}^{\prime}\right] \xi_{\mathrm{P}} \\
& +\frac{\sin \alpha_{\mathrm{P}}}{6 \sin \alpha_{\text {in }}}(E A)_{\text {in }} \kappa_{\text {in }}^{\prime} \xi_{\text {in }}+\left(G D \tau_{\mathrm{P}}+H D \kappa_{\mathrm{P}}^{\prime}\right) \Delta r_{\text {in }}
\end{aligned}
$$

$+\left(G E \tau_{\mathrm{P}}+H E \kappa_{\mathrm{P}}^{\prime}\right) \varepsilon_{0}+0 \cdot \beta_{0}=0$

$(E A)_{\mathrm{st}} \sin \alpha_{\mathrm{st}} \xi_{\mathrm{st}}+\left[6 G B \tau_{\mathrm{P}} \cos \alpha_{\mathrm{P}}+6 H B \kappa_{\mathrm{P}}^{\prime} \cos \alpha_{\mathrm{P}}\right.$

$\left.+6(E A)_{\mathrm{P}} \sin \alpha_{\mathrm{P}}\right] \xi_{\mathrm{P}}+(E A)_{\text {in }} \sin \alpha_{\text {in }} \xi_{\text {in }}$

$+6\left(G D \tau_{\mathrm{P}}+H D \kappa_{\mathrm{P}}^{\prime}\right) \cos \alpha_{\mathrm{P}} \Delta r_{\text {in }}+6\left(G E \tau_{\mathrm{p}}+H E \kappa_{\mathrm{P}}^{\prime}\right)$

$\times \cos \alpha_{\mathrm{P}} \varepsilon_{0}+0 \cdot \beta_{0}=F_{0}-(E A)_{\mathrm{st}} \cos \alpha_{\mathrm{st}} r_{\mathrm{st}} \xi_{\mathrm{st}}$

$+\left[6 H B \sin \alpha_{\mathrm{P}}+6 G B \cos \alpha_{\mathrm{P}}+6(E A)_{\mathrm{P}}\right.$

$\left.\times\left(r_{\text {in }}+r_{\mathrm{P}}\right) \cos \alpha_{\mathrm{P}}-6\left(G B \tau_{\mathrm{P}}+H B \kappa_{\mathrm{P}}^{\prime}\right) r_{\mathrm{P}} \sin \alpha_{\mathrm{P}}\right] \xi_{\mathrm{P}}$

$-(E A)_{\text {in }} \cos \alpha_{\text {in }} r_{\text {in }} \xi_{\text {in }}+\left[6 H D \sin \alpha_{\mathrm{P}}+6 G D \cos \alpha_{\mathrm{P}}\right.$

$\left.-6\left(G D \tau_{\mathrm{P}}+H D \kappa_{\mathrm{P}}^{\prime}\right) r_{\mathrm{P}} \sin \alpha_{\mathrm{P}}\right] \Delta r_{\text {in }}$

$+\left[6 H E \sin \alpha_{\mathrm{P}}+6 G E \cos \alpha_{\mathrm{P}}-6\left(G E \tau_{\mathrm{P}}+H E \kappa_{\mathrm{P}}^{\prime}\right)\right.$

$\left.\times r_{\mathrm{P}} \sin \alpha_{\mathrm{P}}\right] \varepsilon_{0}+0 \cdot \beta_{0}=M_{0}$.

The twist angle $\beta_{0}$ can now be computed from equation system (8). So, the rotation of cable can be evaluated numerically.

\subsection{Numerical results}

Here, the preliminary results obtained on the CFETR CSMC cable are presented. The layout of the CFETR CSMC cable is $\left(2 \mathrm{Nb}_{3} \mathrm{Sn}+\mathrm{Cu}\right) \times 3 \times 4 \times 4 \times 6=864$ strands, containing $576 \mathrm{Nb}_{3} \mathrm{Sn}$ strands and $288 \mathrm{Cu}$ strands. The number of strands in a petal is $3 \times 3 \times 4 \times 4=144$, with $96 \mathrm{Nb}_{3} \mathrm{Sn}$ strands and $48 \mathrm{Cu}$ strands. The diameter of the strands is $0.82 \mathrm{~mm}$. The parameters of the cable are listed in table 1 .

Firstly, the strands inside the cable were assumed to have no slippage. When the cable is getting de-twisted, the subcable is bent and twisted in the opposite direction. The shear force between the petals will change. The relationship between interlayer shear force and de-twisted pitch was calculated with the following equation and the results were 


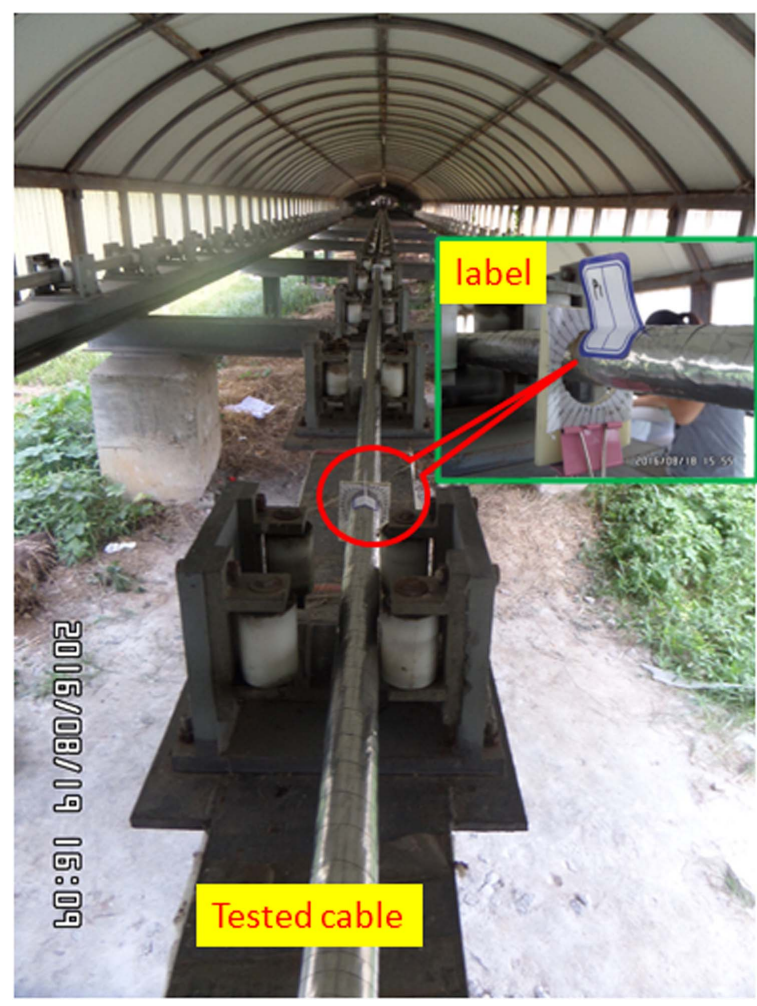

Figure 8. Cable rotation experiment performed on the jacketing line at ASIPP.

shown in figure 5:

$$
F_{n}^{f}(\rho)=F_{n}^{b}-F_{n-1}^{b}=E \pi r^{2} \sin ^{2} \alpha_{24} \frac{R_{n}-R_{n-1}}{\rho} .
$$

The result in figure 5 indicates that the bending stiffness of the petal increases with increased pitch and causes the shear force to increase between the petals.

The petal stiffness can be calculated from the equations given in the annex, which equals to $E I_{\mathrm{eff}}^{\mathrm{Max}}=46.7 \mathrm{~N} \mathrm{~m}^{2}$.

The computation process can be stepwise described as follows:

(1) Obtain the $\beta_{0}$ from the equation system with $E I_{\mathrm{eff}}^{\mathrm{Max}}=46.7 \mathrm{~N} \mathrm{~m}^{2}$;

(2) Obtain the final twist pitch $L_{5}\left(\beta_{0}\right)$ by using $\beta_{0}$;

(3) If $L_{5}\left(\beta_{0}\right)$ is larger than the critical value, it represents $E I_{\text {eff }} \leqslant E I_{\text {eff }}^{\mathrm{Max}}$. It means that the cable will continue to de-twist;

(4) Define $E I_{\text {eff }}=E I$ (if the slip happens at the $n$th layer) and obtain $L_{5}\left(\beta_{0}\right)$. When $L_{5}\left(\beta_{0}\right)$ is smaller than the critical value, the computation is stopped.

In the model, the cabling and wrapping tension were assumed as external forces, which is illustrated in figure 6 .

In figure 6, the insertion force was set to $40 \mathrm{kN}$ and the cabling tension of the petals was set to 200, 400, 600 and $800 \mathrm{~N}$, respectively. The wrapping tension was varied from 50 to 800 N. From figure 6, it appears that the cabling tension has no relevant impact on the cable rotation but the cable rotation decreases distinctly with increased wrapping tension.

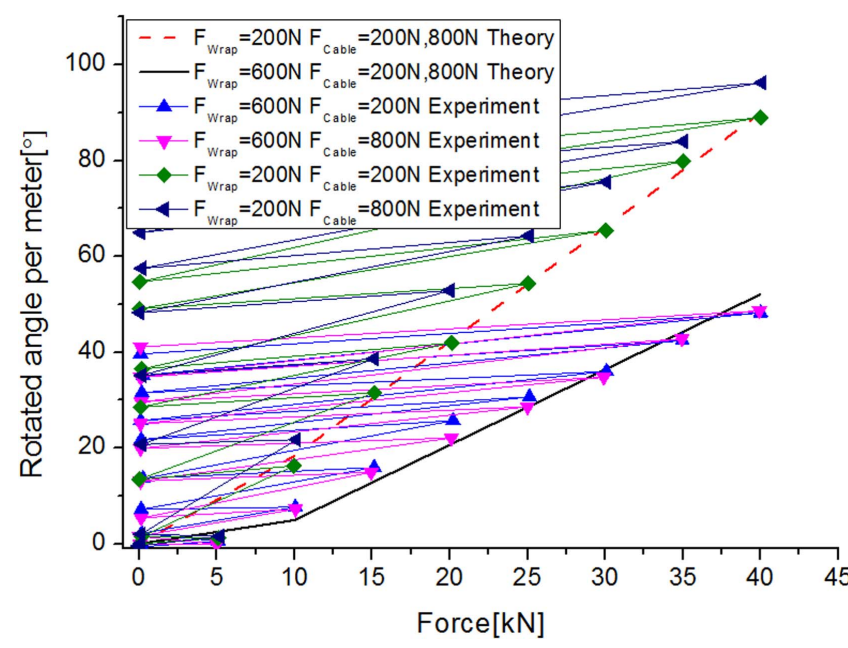

Figure 9. Comparison between numerical and experimental results.

\section{Experimental results and comparison with numerical simulation}

\subsection{Cabling experiments}

Based on the numerical analysis, the cabling experiments with different parameters, as shown in table 2 , have been chosen and performed. In order to reduce the cost, the cables were manufactured with only copper wire.

\subsection{Cable rotation tests}

After the cable manufacturing, the cables were subjected to rotation tests at ASIPP. The experiments were performed at the jacketing line of the ITER conductor workshop. Each cable sample was about $10 \mathrm{~m}$ long. One end of the cable was connected to a wire rope, which is connected to pulling machine and the other end was fixed. Three marks were made on the cable surface at different positions in order to record the rotation during the insertion. The schematic is shown in figure 7 and a picture of the real tested cable is shown in figure 8.

During the experiment, the maximum pulling force was slowly increased from zero until reaching a maximum of $40 \mathrm{kN}$. After reaching the maximum pulling force, the test was stopped.

\subsection{Test results and comparison with numerical modeling}

The experimental and numerical results are compared in figure 9, with remarkable good agreement. Firstly, it is found that the cabling tension has little impact on the cable rotation. Taking the wrapping tension with $200 \mathrm{~N}$ for example, there is no difference between a cabling tension of 200 and $800 \mathrm{~N}$. This experimental result is consistent with the numerical model results.

The rotation is mainly caused by the wrapping tension. The increased wrapping tension reduces the rotation effectively. When the pulling force reaches to $40 \mathrm{kN}$, the rotation angle of cables with $200 \mathrm{~N}$ wrapping tension is about twice of those with $600 \mathrm{~N}$ wrapping tension. 
The analysis based on the numerical model and experimental cable tests provide a solid basis for optimizing the cabling parameters and to reduce the cable rotation. In addition the model can be used to predict the rotation for long cable insertion.

\section{Conclusions}

For large-scale superconducting CICC manufacture for fusion reactors, the cable rotation during jacket insertion appeared to be a problem for the ITER conductors. The large cable rotation could cause a degradation of the conductor performance, which should be kept at a certain value. In order to understand the cause of the rotation and optimize the cabling parameters, a numerical cable model has been built and experimental tests have been performed. Based on the numerical analysis, four CFETR CSMC cable samples with different cabling parameters were manufactured for rotation measurement and analysis. A good agreement between experiments and numerical modeling was achieved. It appears that the cabling tension has a minor influence on the cable rotation but that a higher wrapping tension reduces the cable rotation effectively. The experimental results, analysis and numerical model presented here provide a solid basis for the prediction of the cable rotation for large conductors and consequently an optimization of the cable manufacturing process.

\section{Acknowledgments}

This work is supported by the Fund of Natural Science Foundation of China (No. 51677184, No. 11622217), and National Magnetic Confinement Fusion Science Program (No. 2014GB105001).

\section{Annex}

The symbols used in the annex are listed in table A1.

The cross-section of a petal is illustrated schematically in figure A1. The number of strands in a petal is $3 \times 3 \times$ $4 \times 4=144$, containing $96 \mathrm{Nb}_{3} \mathrm{Sn}$ strands and $48 \mathrm{Cu}$ strands. Firstly, the petal was simplified according to the sequence diagram of strands, as shown in figure A1. The bending central axis and centroid of the petal and geometrical relationship are shown in figure A2. Hence:

The angle of a petal is divided by all strands of $n$th layer.

$$
\theta_{i}=\frac{\pi}{3}+\frac{(2 i-1) \pi}{6 K_{n}}
$$

The expression for the distance between centroid of the petal and $X$-axis is:

$$
y_{C}=\frac{\sum_{n=1}^{13} \sum_{i=1}^{K_{n}} R_{n} \sin \theta_{i}}{144} .
$$

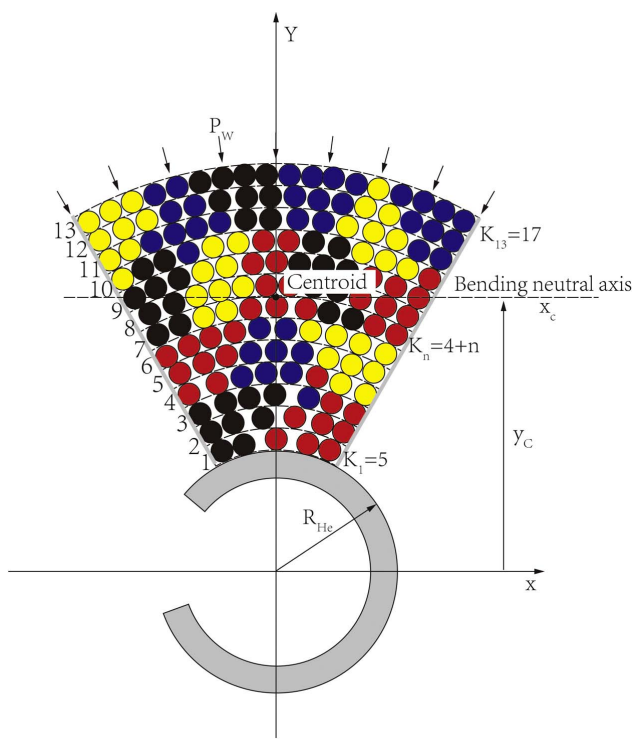

Figure A1. The sequence diagram of strands in a CFETR CSMC petal.

Table A1. List of symbols.

\begin{tabular}{ll}
\hline Symbol & \\
\hline$R_{\mathrm{He}}$ & Inner radius of central cooling spiral \\
$y_{c}$ & Distance between central axis- $x$ and petal centroid \\
$K_{n}$ & Number of strand at $n$th layer \\
$P_{w}$ & Force produced by wrap on petal \\
$R_{n}$ & distance between strands at $n$th layer and conductor \\
& $\quad$ centroid \\
$\theta_{i}$ & Angle between $i$ th strand and $X$-axis \\
$y_{i}$ & Distance between strand at $n$th layer and central cool- \\
& $\quad$ ing tube \\
$\alpha_{24}$ & Angle between center lines of stage 1 and petal \\
$E$ & Young's modulus of strand \\
$r$ & Radius of strand \\
$F_{w}$ & Wrapping tension \\
$R_{\text {Wrap }}$ & Radius of wrap \\
$\alpha_{\text {Wrap }}$ & Helical angle of wrap \\
$L_{\text {Wrap }}$ & Width of wrap \\
\hline
\end{tabular}

If strands do not slide, the effective flexural rigidity of the petal comes to a maximum value. According to the principle of virtual work, it can be expressed as:

$$
\begin{aligned}
E I_{\text {eff }}^{\mathrm{Max}}= & \sum_{n=1}^{13} \sum_{i=1}^{K_{n}} E \pi r^{2}\left[\left(R_{n} \sin \theta_{i}-y_{C}\right)^{2}+\frac{r^{2}}{4}\right] \\
& \times \sin ^{3} \alpha_{24} .
\end{aligned}
$$

If all strands start sliding, the flexural rigidity of a petal is the sum of the flexural rigidity of every strand. It reaches then a theoretical minimum value:

$$
E I_{\mathrm{eff}}^{\mathrm{Min}}=\sum_{n=1}^{13} \sum_{i=1}^{K_{n}} E \frac{\pi r^{4}}{4} \sin ^{3} \alpha_{24}
$$




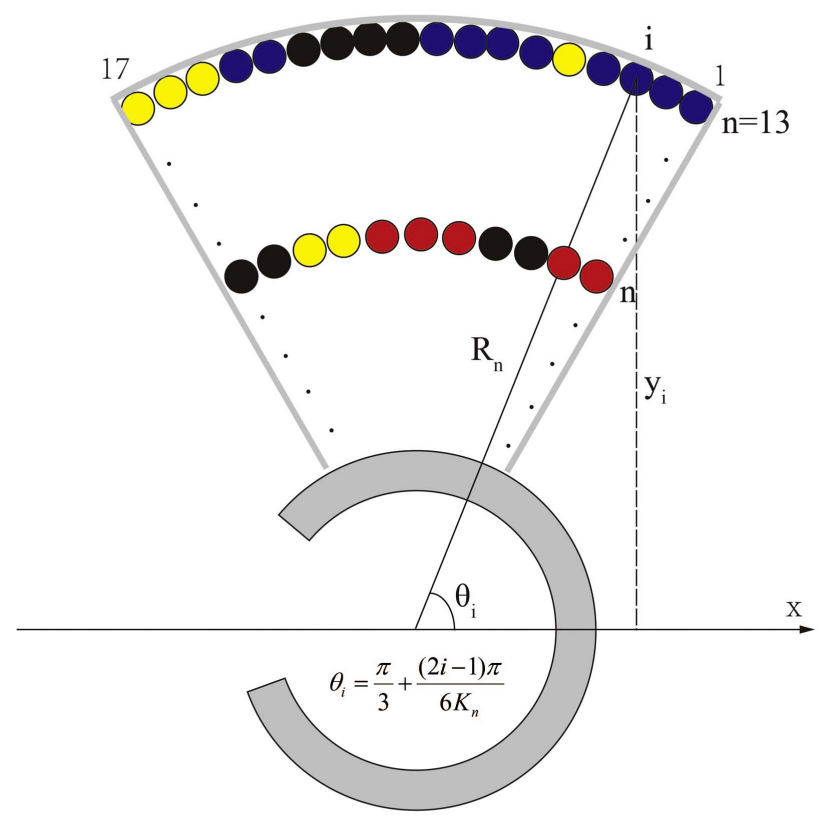

(A)

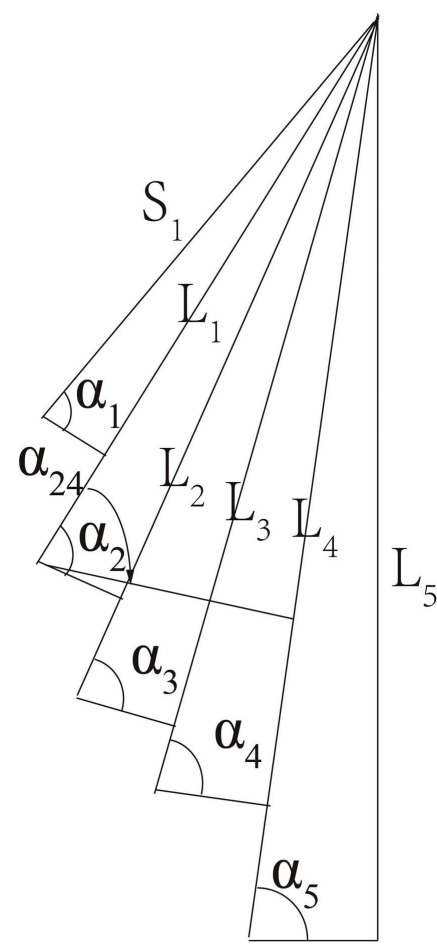

(B)

Figure A2. Geometrical figure ((A): centroid of petal, (B): relationship among different stages).

The formula (6.3) and (6.4) only gives a theoretical maximum value and theoretical minimum value. In fact, the situation is more complex since bending and torsion occurs during the insertion. It has been observed that sometimes only the internal layers of a petal act together while the outer strands could move individually. The flexural rigidity of a sliding section is a super position of the flexural rigidity of single strands. The layer of sliding depends on the radial forces between layers when under bending. Radial forces consist of two parts: one is provided by the wrap; the other is produced by the laying tension in the manufacture and axial tension during the insertion. Radial forces are the largest near the cooling spiral and decrease toward the cable surface. So the outer layer is the first sliding layer. When the curvature of the petal is small, none of the strands slide and the effective flexural rigidity of the petal are given by formula (6.3). With increasing curvature, the shear force between subjacent layers becomes larger and larger. The outermost layer starts sliding when the friction force cannot provide sufficient constraints. In this case, the flexural rigidity of the petal will reduce. With shear force increasing, the second layer and the third layer will start sliding one by one.

When the petal is under bending, the axial force produced by bending on $n$th layer can be expressed as:

$$
F_{n}^{b}=E \pi r^{2} \sin ^{2} \alpha_{24} \frac{R_{n}-y_{C}}{\rho},
$$

where $\rho$ is the radius of curvature of the petal. And similar expressions for the axial produced by bending of $(n-1)$ th layer is:

$$
F_{n-1}^{b}=E \pi r^{2} \sin ^{2} \alpha_{24} \frac{R_{n-1}-y_{C}}{\rho} .
$$

Hence, shear force between $n$th layer and $(n-1)$ th layer can be expressed as:

$$
F_{n}^{f}(\rho)=F_{n}^{b}-F_{n-1}^{b}=E \pi r^{2} \sin ^{2} \alpha_{24} \frac{R_{n}-R_{n-1}}{\rho} .
$$

We assume that the contact force between $n$th and $(n-1)$ th layer is $F^{c}(n)$ and the friction coefficient between wrap and $\mathrm{Cr}$ is $v$. So the maximum friction force can be expressed by $v F^{c}(n)$. Sliding starts when shear force between subjacent layers exceeds friction force. That is described as:

$$
\left(F_{n}^{b}-F_{n-1}^{b}\right) \geqslant \mu F^{C}(n) .
$$

The achievement of criterion equation (6.8) can be proposed to estimate the onset of slippage of strands of $n$th layer against the core formed by inner layers. For the $(n-1)$ th layer, if formula (6.8) can be satisfied, slide will occur. And then transfer to the next layers (e.g. $n-2, n-3, \ldots . .1$ ). So we must calculate the contact force between layers $\left(F^{c}(n)\right)$ in the first place to estimate the number of sliding layer, as shown in figure A3.

The rate of wrap overlap $\eta$ can be expressed as: $\eta=L_{\text {Overlap }} / L_{\text {Wrap }}$.

Hence, the expression of $P_{W}$ is:

$$
P_{W}=\frac{F_{\mathrm{Wrap}}}{L_{\mathrm{Wrap}} R_{\mathrm{Wrap}}}(1+\eta) \cos \alpha_{\mathrm{Wrap}}
$$




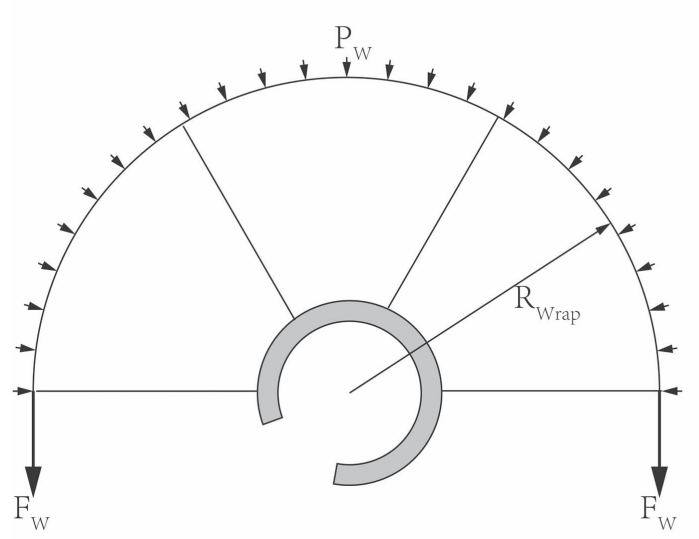

(A)

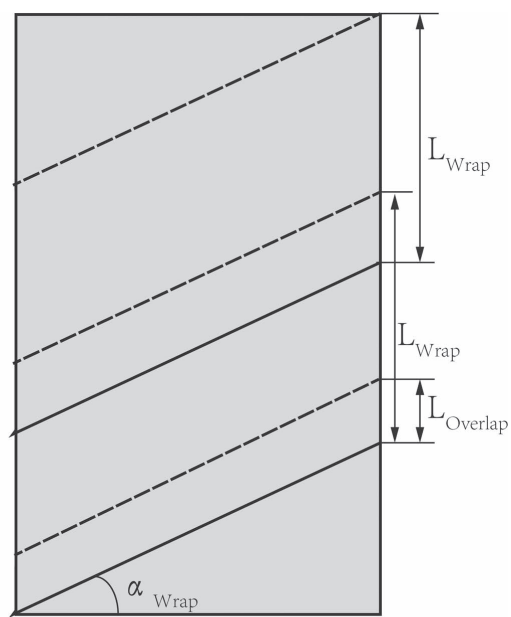

(B)

Figure A3. (A) Radial stress produced by wrapping tension. (B) The rate of wrap overlap.

$F_{\text {Wrap }}^{C}$ is the total stress on the layer $\mathrm{n}$ in a pitch. It can be expressed as:

$$
F_{\mathrm{Wrap}}^{C}(n)=\frac{2 \pi R_{\mathrm{Wrap}} P_{W} L_{1}}{6 K_{n}},
$$

where $L_{1}$ is the pitch of first stage of cabling.

In addition, the radial forces also are produced by laying tension $\left(F_{\text {Cable }}\right)$ during the manufacture and axial tension $\left(F_{\text {Inset }}\right)$ during the insertion. The contact force produced by axial tension on the petal can be calculated approximately by the expression: $F_{j}^{C}=F_{j} / \rho_{j}$. Hence:

$$
\begin{aligned}
F_{\text {cable }}^{C} & +F_{\text {Insert }}^{C} \\
& =\frac{\left(\frac{F_{\text {Insert }}}{6} \sin \alpha_{5}+F_{C \text { able }}\right) \sin \alpha_{4} \sin \alpha_{3} \ldots \sin \alpha_{j}}{144} \frac{S_{1}}{\rho_{j}},
\end{aligned}
$$

where, $\rho_{j}=r_{j} / \cos ^{2} \alpha_{j}$, which is the radius of curvature of $j$ th stage of cabling. $r_{j}$ is the effective radius of $j$ th stage of cabling. And $S_{1}$ is the length of strand of the first stage of cabling in a pitch, which can be expressed as: $S_{1}=$ $2 \pi r_{1} / \cos \alpha_{1}$. In the case of subjecting to axial tension, the contact force produced by the outmost layer on 12th layer in $S_{1}$ can be expressed as:

$$
=\frac{2 \pi \cos \alpha_{1}\left(\frac{F_{\text {Insert }}}{6} \sin \alpha_{5}+F_{\text {Cable }}\right) \sin \alpha_{4} \sin \alpha_{3} \sin \alpha_{2} \sin \alpha_{1}}{144} .
$$

The contact force produced by layer 12 on layer 11 in $S_{1}$ can then be expressed as:

$$
\begin{aligned}
& F_{12}^{C} \\
& =\frac{2 \pi \cos \alpha_{2}\left(\frac{F_{\text {Insert }}}{6} \sin \alpha_{5}+F_{\text {Cable }}\right) \sin \alpha_{4} \sin \alpha_{3} \sin \alpha_{2}}{144} \\
& \times \frac{3 S_{1}}{S_{2}} .
\end{aligned}
$$

The contact force produced by layer 11 on layer 10 in $S_{1}$ can be expressed as:

$$
\begin{aligned}
F_{11}^{C}= & \frac{2 \pi \cos \alpha_{3}\left(\frac{F_{\text {Insert }}}{6} \sin \alpha_{5}+F_{\text {Cable }}\right) \sin \alpha_{4} \sin \alpha_{3}}{144} \\
& \times \frac{9 S_{1}}{S_{3}} .
\end{aligned}
$$

From the above equations, the radial force of $n$th layer $\left(F^{C}(n)\right)$ in the consideration of wrapping tension and axial tension can be expressed as: $F^{C}(n)=F_{n}^{C}+F_{\text {Wrap }}^{C}(n)$.

The geometrical and mechanical parameters of the CFETR CSMC conductor with short twist pitch are shown in table 1. The radius of the $\mathrm{Nb}_{3} \mathrm{Sn}$ and $\mathrm{Cu}$ strand is $0.41 \mathrm{~mm}$ and the radius of the cooling spiral $R_{\mathrm{He}}$ is $5 \mathrm{~mm}$. According to equation (6.2), the distance between the centroid of the petal and $X$-axis is $y_{C}=10.3 \mathrm{~mm}$. The pitches of every stage of cabling are respectively: $L_{1}=22 \mathrm{~mm}, L_{2}=45 \mathrm{~mm}, L_{3}=81 \mathrm{~mm}$, $L_{4}=159 \mathrm{~mm}, L_{5}=443 \mathrm{~mm}$. The effective radii of every stage of cabling are: $r_{1}=0.4629 \mathrm{~mm}, r_{2}=0.9988 \mathrm{~mm}$, $r_{3}=1.6914 \mathrm{~mm}, r_{4}=3.1123 \mathrm{~mm}, r_{5}=10 \mathrm{~mm}$.

The helical angle of every cabling stage is: $\alpha_{1}=$ $82.4691^{\circ}, \alpha_{2}=82.0608^{\circ}, \alpha_{3}=82.5252^{\circ}, \alpha_{4}=82.9885^{\circ}$, $\alpha_{5}=81.9274^{\circ}$. The angle between the center line of the first stage of cabling and the center line of a petal $\alpha_{24}=67.571^{\circ}$. The width of the wrap $L_{\mathrm{Wrap}}$ is $40 \mathrm{~mm}$. The radius of the wrap $R_{\mathrm{Wrap}}$ is $15.5 \mathrm{~mm}$ and the rate of wrap overlap $\eta$ is $40 \%$. The pitch of wrap is $24 \mathrm{~mm}$ and the helical angle $\alpha_{\text {Wrap }}$ is $13.8437^{\circ}$. Wrapping tension consists of two situations including $F_{W}=200 \mathrm{~N}$ and $F_{W}=600 \mathrm{~N}$. The cabling tension also consists of two situations including $F_{\text {Cable }}=200 \mathrm{~N}$ and $F=800$ $\mathrm{N}$. The maximum tension during the insertion $F_{\text {Insert }}$ is $40 \mathrm{kN}$. The radial stress on the 10th-13th layer is shown in table A2.

According to equation (6.8), with the shear force between subjacent layers increasing, the number of sliding layers increases gradually while the flexural rigidity of the petal decreases gradually. The results of the calculations are shown 


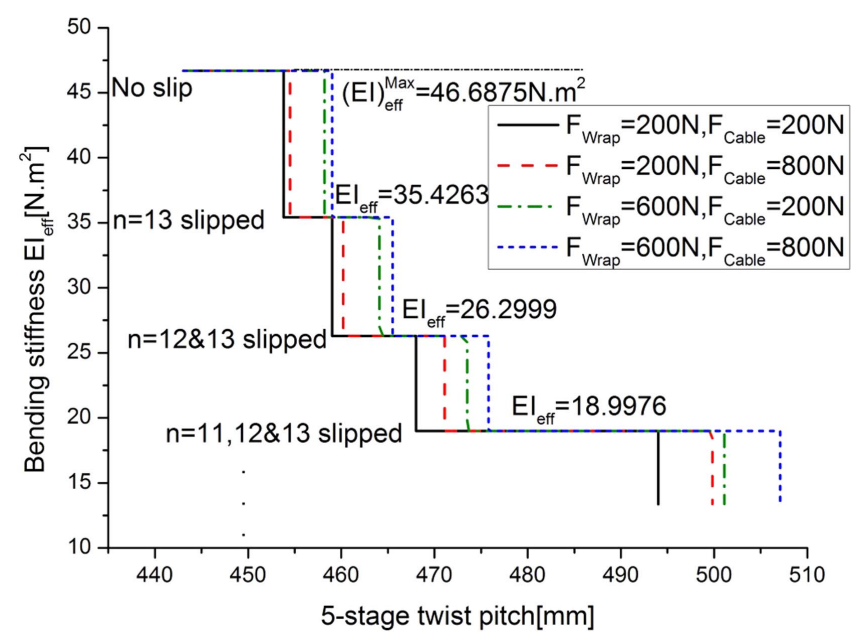

Figure A4. The relationship between flexural rigidity of the petal and pitch of the fifth cabling stage after untwisting.

Table A2. Cabling experiment with different parameters.

\begin{tabular}{lcc}
\hline Sample & Final cabling tension $(\mathrm{N})$ & Final wrapping tension $(\mathrm{N})$ \\
\hline CS-1 & 200 & 600 \\
CS-2 & 800 & 600 \\
CS-3 & 200 & 200 \\
CS-4 & 800 & 200 \\
\hline
\end{tabular}

in figure A4 illustrating the variation in $E I$ as a function of cabling pitch of the fifth stage.

During untwisting, the cable pitch increases, which reduces the flexural rigidity of the petal step by step. With increased degree of untwisting, the curvature of the petal increases. In addition, the shear force between subjacent layers also increases. Hence, the number of sliding layers increases, which reduces the flexural rigidity of the petal step by step. We also find that larger wrapping and cabling tension can increase the flexural rigidity of the petal and impede the degree of untwisting.

\section{Disclaimer}

The views and opinions expressed herein do not necessarily reflect those of the ITER Organization.

\section{ORCID iDs}

Jinggang Qin (10) https://orcid.org/0000-0002-5652-3447

\section{References}

[1] Wan Y 2012 Mission of CFETR ITER Training Forum and 2nd Workshop on MFE Development Strategy (Hefei, 6 September)
[2] Song Y et al 2014 Concept design of CFETR tokamak machine IEEE Trans. Plasma Sci. 42 503-9

[3] Li J et al 2015 Chinese program on CFETR SOFE 2015 (Austin TX, USA, 31 May-4 June)

[4] Devred A et al 2014 Challenges and status of ITER conductor production Supercond. Sci. Technol. 27044001

[5] Qin J et al 2017 Manufacturing of $\mathrm{Nb}_{3} \mathrm{Sn}$ sample conductor for CFETR central solenoid model coil IEEE Trans. Appl. Supercond. 274200805

[6] Wu Y et al 2016 Status of the ITER conductors in China IEEE Trans. Appl. Supercond. 266000405

[7] Devred A, Backbier I, Bessette D, Bevillard G, Gardner M, Jewell M, Mitchell N, Pong I and Vostner A 2012 Status of ITER conductor development and production IEEE Trans. Appl. Supercond. 224804909

[8] Wu Y et al 2013 Manufacturing of the ITER TF conductors in China IEEE Trans. Appl. Supercond. 234802004

[9] Di Zenobio A et al 2012 Conductor manufacturing of the ITER TF full-size performance samples IEEE Trans. Appl. Supercond. 20 1412-5

[10] Zani L et al 2016 Overview of progress on the EU DEMO reactor magnet system design IEEE Trans. Appl. Supercond. 264204505

[11] Kim K et al 2015 Conceptual design study of the K-DEMO magnet system Fusion Eng. Des. 96/97 281-5

[12] Painter T A et al 2015 Cable untwisting and final-stage twist pitch measurements of ITER toroidal field $\mathrm{Nb}_{3} \mathrm{Sn}$ cable-inconduit conductors IEEE Trans. Appl. Supercond. 25 4800604

[13] Nabara Y et al 2015 Behavior of $\mathrm{Nb}_{3}$ Sn cable assembled with conduit for ITER central solenoid IEEE Trans. Appl. Supercond. 254200305

[14] Takahashi Y et al 2013 Cable twist pitch variation in $\mathrm{Nb}_{3} \mathrm{Sn}$ conductors for ITER toroidal feld coils in Japan IEEE Trans. Appl. Supercond. 234801504

[15] Kaverin D S et al 2014 VNIIKP RF TF cable untwisting and elongation under tensile force IEEE Trans. Appl. Supercond. 244801104

[16] Nijhuis A and Ilyin Y 2006 Transverse load optimization in $\mathrm{Nb}_{3}$ Sn CICC design; influence of cabling, void fraction and strand stiffness Supercond. Sci. Technol. 19 945-62

[17] Takahashi Y et al 2001 AC loss measurement of $46 \mathrm{kA}-13 \mathrm{~T}$ $\mathrm{Nb}_{3} \mathrm{Sn}$ conductor for ITER IEEE Trans. Appl. Supercond. 11 1546-9

[18] Stepanov B, Bruzzone P, Sedlak K and Wesche R 2014 Recent tests of the ITER conductors and highlights in $\mathrm{Nb}_{3} \mathrm{Sn}$ conductors behavior IEEE Trans. Appl. Supercond. 24 4802105

[19] Qin J et al 2015 Cable rotation and twist pitch variation for ITER TF conductor in China IEEE Trans. Appl. Supercond. 258800204

[20] Bessette D 2013 Cabling and jacketing Conductor Meeting (Italy) 09.30-10.02

[21] Qin J et al 2015 Optimization of CFETR CSMC cabling based on numerical modeling and experiments Supercond. Sci. Technol. 28125008

[22] Costello G A 1997 Theory of Wire Rope (New York: Springer)

[23] Lanteigne J 1985 Theoretical estimation of the response of helically armored cables to tension, torsion, and bending ASME J. Appl. Mech. 52 423-32

[24] Qin J, Wu Y, Warnet L L and Nijhuis A 2011 A novel numerical mechanical model for the stress-strain distribution in superconducting cable-in-conduit conductors Supercond. Sci. Technol. 24065012 